\title{
Coronary Sinus Aneurysm: Incidental discovery during coronary artery bypass grafting
}

\author{
Arjun Balakumar ${ }^{1}$, Fahad Athar ${ }^{2}$ and Louis Samuels ${ }^{3 *}$ \\ ${ }^{1}$ Sidney Kimmel Medical College of Thomas Jefferson University, Philadelphia, PA, USA \\ ${ }^{2}$ Albert Einstein Medical Center, Philadelphia, PA, USA \\ ${ }^{3}$ Department of Surgery, Division of Cardiothoracic Surgery, Thomas Jefferson University, Philadelphia, PA, USA
}

\begin{abstract}
The case of a 68-year-old man with symptomatic coronary artery disease undergoing coronary artery bypass grafting was incidentally found to have a coronary sinus (CS) aneurysm at the time of surgery. The aneurysm itself did not impact the operation but raised questions about associated anomalies and whether there was a need for operative management. This report describes the details of the case as well as reviews the information known to date regarding CS aneurysms.
\end{abstract}

\section{Introduction}

Coronary venous development and its variants are topics of interest for the anatomist, embryologist, and pathologist. In contrast to coronary arterial pathology, coronary venous abnormalities are less clinically relevant. However, on occasion, the incidental or deliberate discovery of a coronary venous anomaly may represent a direct problem or indicate a possible association with another condition. In addition, the presence of a coronary venous aneurysm may pose technical problems in certain procedures. The cardiothoracic surgical literature is limited with respect to coronary venous pathologies, particularly in the adult. We report the case of a 68 -year-old man who was found to have a coronary venous aneurysm at the confluence of the great cardiac vein (GCV) and coronary sinus (CS) during coronary artery bypass grafting.

\section{Case}

A 68-year-old man with a past medical history significant for coronary artery disease (CAD), hypertension, hyperlipidemia, and myocardial infarction with previous percutaneous coronary intervention presented to the cardiology clinic with exertional chest pain. Additional non-cardiovascular conditions included emphysema, asthma, and gastro-esophageal reflux disease with erosive esophagitis, hypothyroidism, and occasional dizziness/lightheadedness with falls. There was no history of arrhythmia or congenital abnormalities. The social history included a 75-pack year smoking, daily marijuana use, no alcohol use, and worked as a heavy machine operator. Home medications were aspirin, clopidogrel, carvedilol, fenofibrate, isosorbide mononitrate, losartan, escitalopram, and levothyroxine. $\mathrm{He}$ was referred for ischemic evaluation via left heart catheterization.

Catheterization showed in-stent stenosis of the left anterior descending coronary artery (LAD) with total occlusion of the first obtuse marginal branch of the left circumflex artery. Echocardiography showed normal left and right ventricular size and function with some hypokinesis of the inferior wall. There was no valvular pathology or congenital anomalies. The electrocardiogram showed a normal sinus rhythm with a left anterior fascicular block. He was referred to cardiothoracic surgery for coronary artery bypass grafting (CABG) consideration.

The CABG procedure was conducted via a median sternotomy utilizing a pump-assisted beating heart technique under normothermic conditions. Standard aortic and right atrial cannulation was utilized. Inspection of the heart revealed a large coronary venous aneurysm (Figure 1) on the inferior wall corresponding to the confluence of the GCV and the CS in the territory of the posterior descending artery (PDA). The aneurysm measured approximately $5 \mathrm{X} 4 \mathrm{X} 4$ centimeters. Fortunately, the PDA did not require bypass grafting since the aneurysm obscured most its view. The surgery continued with a saphenous vein graft (SVG) to the obtuse marginal branch of the left circumflex artery and a left internal mammary (LIMA) bypass to the LAD. While the case was taking place, a literature search for coronary venous aneurysms encountered during cardiac surgery was conducted - limited information was found. As such, the anomaly was left alone. The procedure was completed without complication. The postoperative course was unremarkable, and the patient discharged in stable condition.

\section{Discussion}

The coronary venous system is an elaborate array of vascular tributaries that ultimately drain into the coronary sinus (Figure 2). The high degree of coronary venous branch variability has occasionally created problems and obstacles for the clinician, particularly those in which certain procedures depend upon specific anatomic considerations (e.g. LV electrode placement for bi-ventricular pacing, CS cardioplegia catheter placement for myocardial protection during open-heart surgery). In 2005, Singh and others, in an effort to facilitate electrophysiologic procedures, published a thorough description of the

${ }^{*}$ Correspondence to: Louis Samuels, Department of Surgery, Division of Cardiothoracic Surgery, Thomas Jefferson University, Philadelphia, PA, USA, E-mail: samuelsle@aol.com

Received: October 12, 2018; Accepted: October 20, 2018; Published: October 25, 2018 
coronary venous system [1]. Together with cardiac imaging, the authors provided a systematic and segmental classification of coronary venous anatomy. Notably absent were coronary venous aneurysms.

An extensive review of coronary veins along with an anatomic classification based on Computer Tomographic (CT) and Magnetic Resonance Imaging (MRI) was provided by Saremi and colleagues in 2015 [2]. This publication provided excellent illustrations and imaging of coronary venous anatomy and pathology, offering great details on normal and variants of the coronary venous system. In addition, a discussion of the clinical associations of coronary venous pathology was provided, highlighting conduction system abnormalities as well as noting the importance of imaging for electrophysiologic interventions.

Clinically relevant reports of coronary venous aneurysms have been mostly associated with ventricular arrhythmia and the concern for sudden cardiac death. In 1988, Ho and colleagues reported the fourth case of a coronary venous aneurysm associated with an accessory pathway [3]. In this report, a 12-year-old girl with a history of neonatal arrhythmia died suddenly. Histologic examination of the necropsy heart showed myocardial abnormalities that likely contributed to aberrant conduction predisposing to a lethal arrhythmia. These findingsand review of the three cases preceding this one-- lead the authors to express their concern that coronary sinus or venous aneurysm may not be as benign as previously thought.

In adults, the literature related to coronary venous aneurysms is scant. In 2007, Loukas et al. reported an aneurysm of the great cardiac vein in an 87-year-old man (Figure 3). This anomaly appeared to be due to a possible distal constriction of the great cardiac vein by a small muscular branch of the circumflex branch and a possible proximal constriction by the left marginal artery [4]. In 2010, a retrospective study of 187 adult patients undergoing CT angiography for CAD was undertaken with the aim of determining the prevalence of coronary venous aneurysms with no history of cardiac arrhythmias [5]. A single
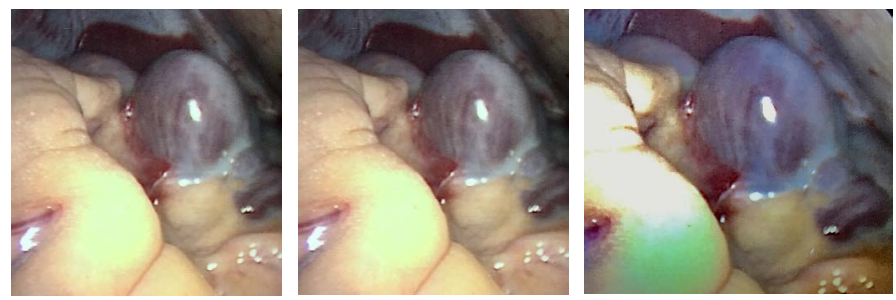

Figure 1. Coronary sinus aneurysm—intraoperative photograph

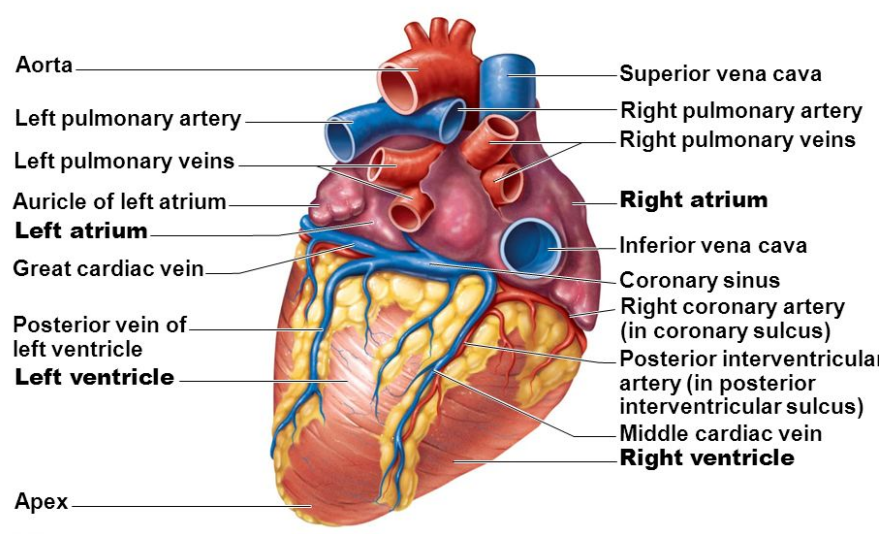

(d) Posterior surface view

Figure 2. Coronary venous anatomy

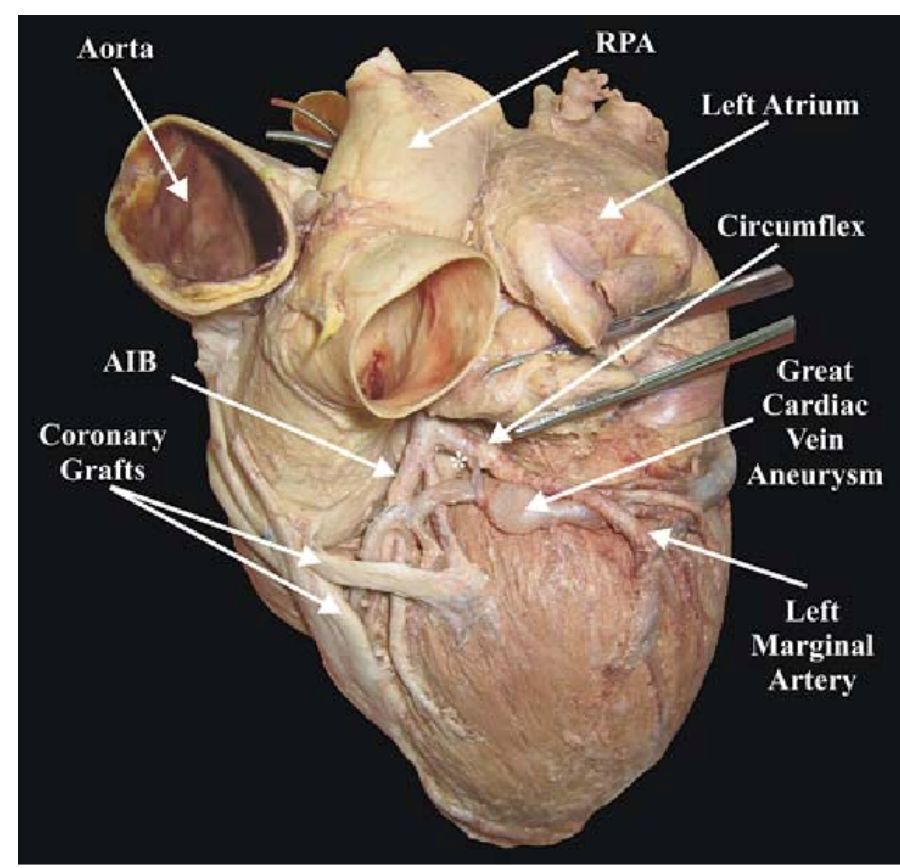

Figure 3. Aneurysm of the great cardiac vein

From: Loukas M, Tubbs RS, Jordan R (2007) Aneurysm of the great cardiac vein. Surg Radiol Anat 29: 169-72. [Crossref]

aneurysm was found in $19(10 \%)$ patients. The most common location was the posterior interventricular vein near the confluence with the coronary sinus and the majority were fusiform. There were only 3 diverticular aneurysms and 3 at the junction of the GCV and CS, as was seen in our case. Finally, in 2017, Song and colleagues described the first case of three co-existing systemic vein anomalies in a 67-yearold woman undergoing CABG surgery [6]. A preoperative chest CT scan with contrast demonstrated interruption of the left IVC, persistent left SVC, and an anomalous left hepatic vein draining into a coronary sinus aneurysm. The CABG procedure confirmed the anomalies and was otherwise uneventful.

Based on cardiac imaging and anecdotal reports, several comments can be made regarding coronary venous aneurysms: 1) the incidence is at least $10 \%$ based on the report by Saremi et al. [5]; 2) there is an association with cardiac arrhythmia and other venous anomalies in some cases [3,6]; 3) clinicians involved with surgical or interventional procedures should be mindful of these anomalies since they may impact the ability to safely access the coronary venous system for such things as retrograde catheters to deliver cardioplegia or placement of LV epicardial leads.

In summary, this case report adds to the experience of coronary venous aneurysm in the setting of CABG surgery. Knowledge of this entity may have technical and clinical implications.

\section{References}

1. Singh JP, Houser S, Heist EK, et al. (2005) The coronary venous anatomy. J Am Coll Cardiol 46: 68-74.

2. Saremi F, Muresian H, Sanchez-Quintana D (2012) Coronary Veins: Comprehensive CT-Anatomic Classification and Review of Variants and Clinical Implications. Radiographics 32: E1-E32. [Crossref]

3. Ho, SY, Russell G, Rowland E (1988) Coronary venous aneurysms and accessory atrioventricular connections. Br Heart J 60: 348-51. [Crossref]

4. Loukas M, Tubbs RS, Jordan R (2007) Aneurysm of the great cardiac vein. Surg Radiol Anat 29: 169-72. 
Balakumar A (2018) Coronary Sinus Aneurysm: Incidental discovery during coronary artery bypass grafting

5. Saremi F, Channual S, Sarlaty T, Tafti MA, Milliken JC, et al. (2010) Coronary Venous Aneurysm in Patients Without Cardiac Arrhythmia as Detected by MDCT. J Am Coll Cardiol Img 3: 257-65. [Crossref]
6. Song G, Du M, Ren W, Zhou K, Sun L (2017) Coronary sinus aneurysm associated with multiple venous anomalies. BMC Cardiovasc Disord 17: 95. [Crossref]

Copyright: $\odot 2018$ Balakumar A. This is an open-access article distributed under the terms of the Creative Commons Attribution License, which permits unrestricted use, distribution, and reproduction in any medium, provided the original author and source are credited. 